\title{
Regulation of salt appetite and blood pressure
}

Loss-of-function mutations in $H S D 11 B 2$, which encodes the glucocorticoid-metabolizing enzyme $11 \beta \mathrm{HSD} 2$, result in unregulated activation of the mineralocorticoid receptor (MR) by endogenous glucocorticoid and development of the hypertensive syndrome apparent mineralocorticoid excess. Now, researchers report that in the brain, 11ßHSD2 has a role in the control of salt appetite and the blood-pressure response to dietary sodium. They suggest that $11 \beta$ HSD2-positive neurons integrate salt appetite and salt sensitivity through a MR-dependent pathway.

Matthew Bailey and colleagues previously found that global knockout of $H s d 11 b 2$ in mice results in hypertension and reduced renal sodium excretion. They now show that brain-specific knockout of this gene does not affect basal blood pressure in mice, but results in a threefold increase in salt appetite compared with littermate controls and induces salt sensitivity of blood pressure.

"Deleting Hsd11b2 in the brain generates a strong and physiologically unnecessary behavioural drive to ingest salt," says Bailey. "This result is interesting and builds on previous work that associated Hsd11b2-expressing neurons with salt-seeking behaviour. Our major finding is that when salt is eaten the knockout mice get high blood pressure; removing the salt lowers blood pressure. Control mice don't respond in this way."

When knockout and control mice were fed the same high-salt diet, only the knockout mice became hypertensive. Moreover, urinary sodium excretion did not differ between the two groups, suggesting that the effect of brain-specific $H s d 11$ b2 deletion on blood pressure was independent of the kidney. The knockout mice did, however, show evidence of impaired autonomic cardiac control and an attenuated baroreflex. Bailey explains that rather than affecting renal salt excretion, deletion of $\mathrm{Hsd} 11 \mathrm{~b} 2 \mathrm{in}$ the brain seems to change the way in which blood vessels contract and the heart responds to changes in blood pressure; the mechanism by which these effects occur is not yet known.

"In a broad context, the pathways that control salt hunger in the brain also control salt taste perception by the tongue, salt uptake by the gut and salt excretion by the kidney," says Bailey. "This integration makes evolutionary sense, but the environment

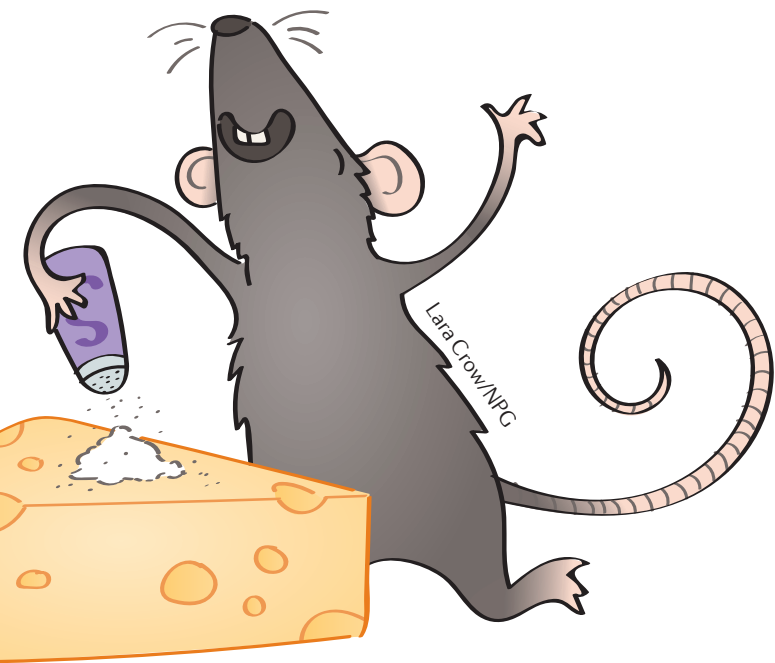

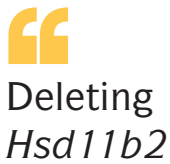

in the brain generates a strong and physiologically unnecessary behavioural drive to ingest salt has changed - salt is no longer scarce and mutations that alter how these pathways function can give us multiple hits for salt-induced hypertension."

He suggests that the new findings might have specific clinical implications for certain patient groups, such as those with heart or kidney failure who have to reduce their salt intake but find this restriction very difficult. In particular, he notes that treatment with the MR antagonist spironolactone reduced the salt appetite of the knockout mice and might potentially have a similar effect in patients. As well as continuing their investigations in mice, the researchers are currently conducting a small clinical study to assess salt intake and perception in patients with heart failure.

Ellen F. Carney

ORIGINAL ARTICLE Evans, L. C. et al. Conditional deletion of $H s d 11 b 2$ in the brain causes salt appetite and hypertension. Circulation http:// dx.doi.org/10.1161/CIRCULATIONAHA.115.019341 